\title{
Neuroscience Robotics to Investigate Multisensory Integration and Bodily Awareness
}

\author{
J. Duenas, D. Chapuis, C. Pfeiffer, R. Martuzzi, S. Ionta, O. Blanke and R. Gassert
}

\begin{abstract}
Humans experience the self as localized within their body. This aspect of bodily self-consciousness can be experimentally manipulated by exposing individuals to conflicting multisensory input, or can be abnormal following focal brain injury. Recent technological developments helped to unravel some of the mechanisms underlying multisensory integration and self-location, but the neural underpinnings are still under investigation, and the manual application of stimuli resulted in large variability difficult to control. This paper presents the development and evaluation of an MR-compatible stroking device capable of presenting moving tactile stimuli to both legs and the back of participants lying on a scanner bed while acquiring functional neuroimaging data. The platform consists of four independent stroking devices with a travel of $16-20 \mathrm{~cm}$ and a maximum stroking velocity of $15 \mathrm{~cm} / \mathrm{s}$, actuated over non-magnetic ultrasonic motors. Complemented with virtual reality, this setup provides a unique research platform allowing to investigate multisensory integration and its effects on selflocation under well-controlled experimental conditions. The MR-compatibility of the system was evaluated in both a 3 and a 7 Tesla scanner and showed negligible interference with brain imaging. In a preliminary study using a prototype device with only one tactile stimulator, fMRI data acquired on 12 healthy participants showed visuo-tactile synchrony-related and body-specific modulations of the brain activity in bilateral temporoparietal cortex.
\end{abstract}

\section{INTRODUCTION}

With the rapid development and availability of functional brain imaging techniques, there has been an increasing interest in unraveling the mechanisms underlying multisensory integration and distortions associated with multisensory conflicts or brain injury. Of particular interest for the present research are neurological patients with out-of-body experiences (OBE) who suffer from abnormal self-location due to a distortion of multisensory integration after damage to the temporoparietal cortex [1]. Although self-location can be studied experimentally [2], the neural underpinnings of self-location have yet to be investigated and protocols from cognitive science need to be adapted to such experimental environments (Fig 1).

Earlier studies investigated mechanisms of the bodily self in healthy participants by using visuo-tactile conflicts during the so-called rubber hand illusion, in which manual stroking was applied to both the participant's and a rubber hand [3]. Virtual reality (VR) technology has allowed to expand this paradigm through the application of multisensory conflicts to

J. Duenas, D. Chapuis and R. Gassert are with the Rehabilitation Engineering Lab, ETH Zurich, Switzerland.

J. Duenas, C. Pfeiffer, R. Martuzzi, S. Ionta and O. Blanke are with the Laboratory of Cognitive Neuroscience, EPFL, Lausanne, Switzerland.

Corresponding author's email: gassertrdethz.ch

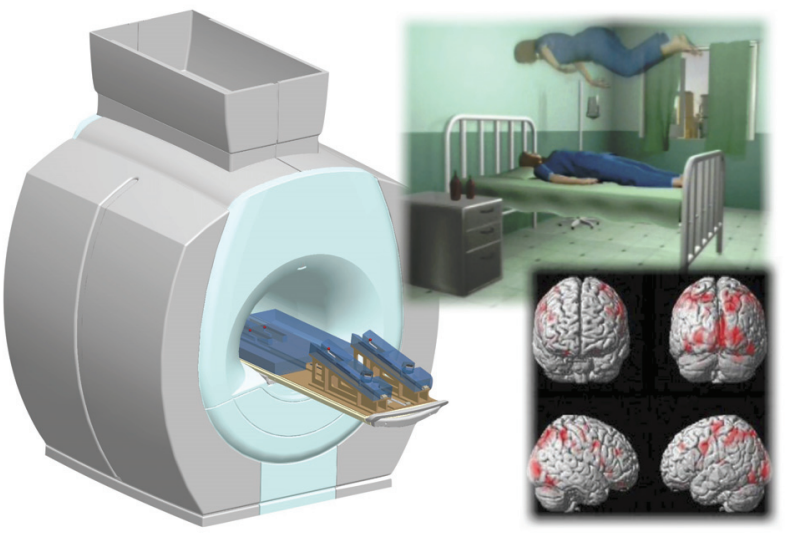

Fig. 1: Neuroscience robotics: investigating the neural correlates of multisensory integration and bodily awareness with neuroimaging, robotics and virtual reality.

the whole body, and investigating the underlying mechanisms in a more controlled manner, e.g. by projecting a virtual body in front of the participant and presenting synchronous and asynchronous visuo-tactile stimuli to the real and virtual body [4], [5]. Importantly, these studies induced errors in self-location with predictable patterns towards the fake or virtual body. While experiments focusing on the arm have been extended to brain imaging studies [6], [7], this has not been the case for full body illusions (FBI). Further, in all these studies, tactile stimuli were applied manually, resulting in large variability as well as limited control over the experimental conditions and the resulting effects.

Robotic systems are ideal tools to create precise and repeatable visuo-tactile conditions and promise further improvements in experimental control in virtual environments [8]. As we are interested in investigating the neural mechanisms of multisensory integration and self-location, it is important to also have a window onto the brain. Functional magnetic resonance imaging (fMRI) is an ideal research tool for this endeavor, providing high spatial resolution and whole brain coverage, and being widely available. Robotic systems compatible with this neuroimaging technique have been proposed to investigate sensorimotor control and learning [9]-[11]. The combination of fMRI, neuroscience robotics and virtual reality now promises the ability to manipulate and assess sensorimotor states of the participants while investigating the underlying neural mechanisms [8], in order to gain new insights into multisensory integration and the consequences of sensory conflicts on specific brain mechanisms 
[12]. However, to date there have only been very few studies on multisensory integration combining all three technologies (robotics, virtual reality and neuroimaging). In this paper we present an MR-compatible stroking device capable of applying moving tactile stimuli in a well-controlled and repeatable manner to the back and legs of a participant lying supine in an MR scanner. Four independent modules are integrated into a platform that is covered by an ergonomic mattress, which fits into the bed of the MR system. A preliminary study using a single stroking module on the back of participants allowed inducing illusory changes in selflocation and related aspects as described in [2] in an MR environment.

The structure of this paper is as follows: Section II details the design of the system, Section III describes its dynamic performance and the MR compatibility testing. Section IV presents a preliminary study to induce changes in selflocation and the associated brain activity. Finally, Section V summarizes our conclusions.

\section{CONCEPT}

\section{A. Requirements}

\section{- MR Safety and Compatibility}

The magnetic resonance (MR) environment imposes strong safety and electromagnetic compatibility constraints for robotic systems to be used in the proximity of the MRI bore [10], [13]. The high magnetic field $(3 T-7 T$ in our case) and associated spatial gradient prevents the use of ferromagnetic components. In addition, the powerful radiofrequency pulses used in MR imaging, could result in mutual interference. At the same time, any electromagnetic noise emitted by the system could disturb the sensitive imaging.

A crucial requirement for achieving MR compatibility is the correct choice of the actuation system. Most MRIcompatible robots presented to date were actuated by ultrasonic motors, or use conventional actuators situated outside the MR room combined with mechanical, pneumatic or hydraulic transmissions [14]. The presented system is actuated over four non-magnetic ultrasonic motors (USM), one per stroking module. The device contains two modules located at the level of the participant's back (back modules, $\mathrm{BM}$ ) and two modules located below the legs (leg modules, LM). Shielded cables and D-sub radio frequency (RF) filters link the USMs to the control box placed outside MR room. The control box contains USM drivers, logic to control the motors according to specifications and a safety circuit (Fig. 2). To improve compatibility, the USMs and encoders are located in the lower third of the scanner bed, to place them as far away from the region of interest (i.e. the brain) as possible.

\section{- Workspace and Ergonomics}

The limited workspace inside the scanner bore imposes constraints on the development of the robotic system. The designed system must therefore fit onto the scanner bed without significantly elevating the trunk and head of the

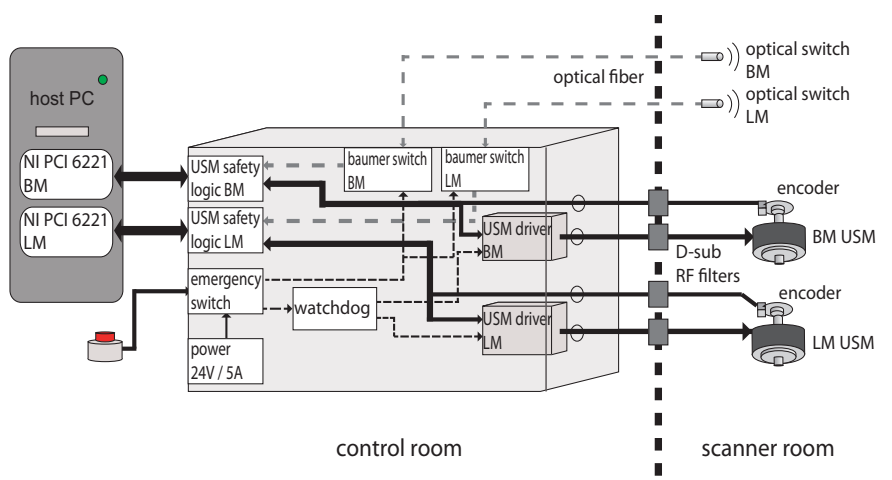

Fig. 2: Schematic of the actuation system. The control box is located outside of the MR room, in the control room, and linked to the motors over shielded cables and RF filters integrated into the penetration panel. Fiberoptical switches are used to initialize the four modules.

participant (both for comfort and image quality), and provide a comfortable and ergonomic support. For this reason, the four modules are fixed to a flexible wooden board which replaces the normal mattress of the scanner bed. Each of the modules is covered by a CNC machine-cut multi-layer mattress, consisting of a medium-strength core that follows the shape of the body and is covered with a soft cushion. The leg modules can be adjusted to the height of the participant and are inclined to prevent discomfort in the knee joints. The system therefore nicely fits into the scanner bore and provides a comfortable posture (Fig. 3).

\section{B. Stroking Mechanism}

In previous studies stimuli were often applied manually by an experimenter, resulting in variability of range, amplitude and phase shift which are difficult to control. Tactile stimuli were delivered according to the standard procedure established in [2], [4], [5], [15], based on an approach that was adapted and extended from the original rubber hand procedure (reviewed in [16]). The most critical point in this procedure is the synchrony between tactile and visual stimuli, which motivated the introduction of a robotic stimulator and virtual reality environment allowing to greatly increase the control over experimental conditions and possible variety [8].

The stroking mechanism (Fig. 4) consists of four individual stimulation modules actuated by four USMs on a linear movement $(l)$ generated by a rack-and-pinion mechanism. The stimulation module consists of a polymer sphere fixed to a polymer spring blade (EP GC 203, of $1 \mathrm{~mm}$ thickness, Angst and Pfister). The blade assures a constant contact pressure while the sphere follows the shape of the back. The sphere-blade assembly is attached to a mobile base on a linear guide, which limits the maximum deflection $(\alpha)$ of the blade.

\section{Virtual Reality Feedback}

The virtual representation of the body being stimulated is crucial to induce OBE. The visual feedback is presented through a head mounted display, and shows the back of a 
a)

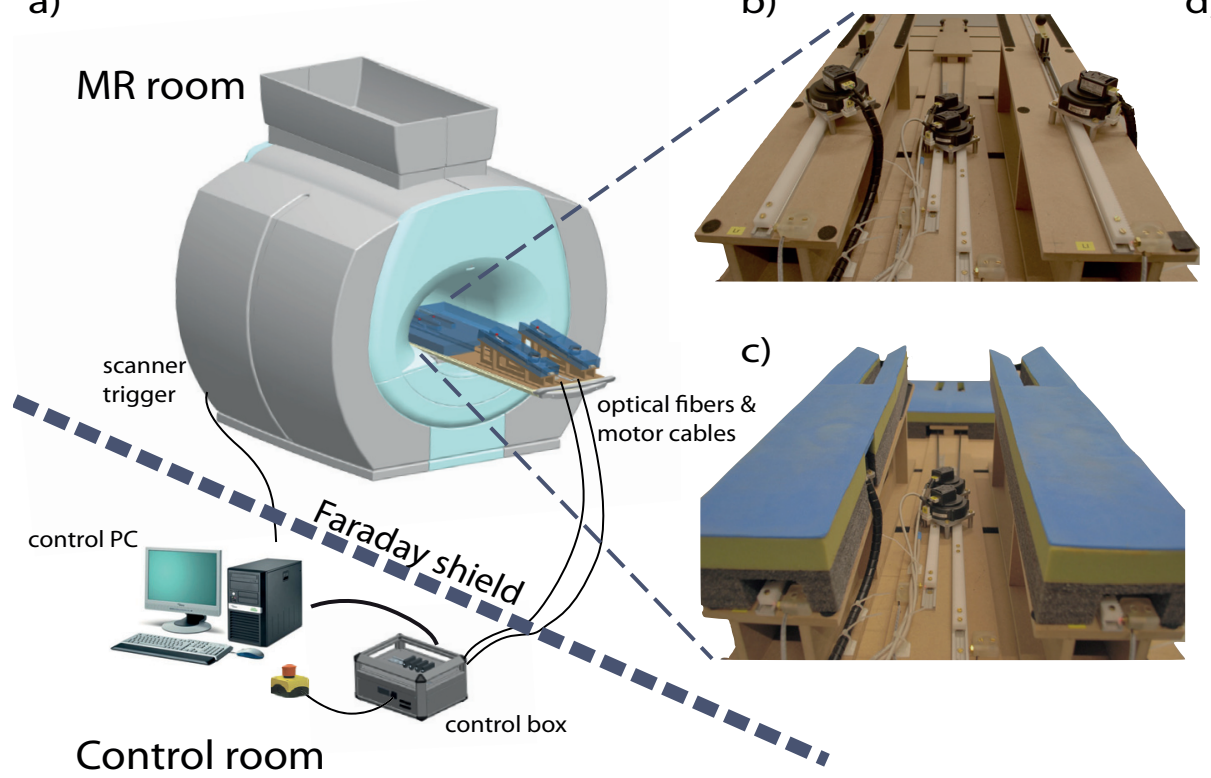

d)

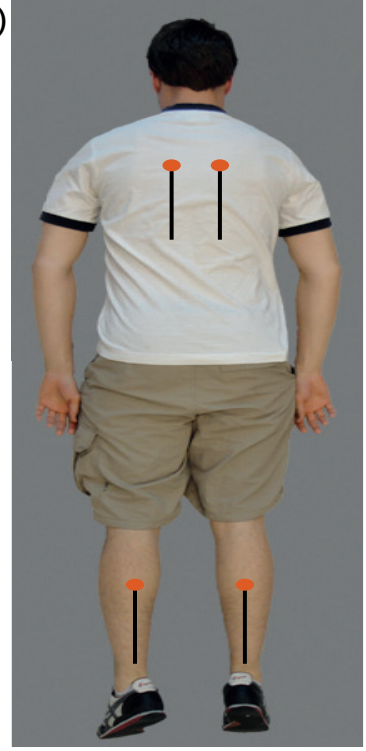

Fig. 3: The fMRI-compatible stroking device consists of four individual stimulation modules. a) Placement of the device in an MR environment, on the scanner bed. b) Device base structure with ultrasonic motors, linear guides and stroking spheres. c) Complete device covered with ergonomic mattress. d) Virtual reality display showing the four virtual moving stimuli.

body hovering about $2 \mathrm{~m}$ above the participants body. The stroking modules are represented by four individual moving dots rendered over the picture of the body (Fig. 3d). This virtual reality setup provides full control over the visual feedback, allowing the introduction and manipulation of delays to present the visual stimuli in synchrony or asynchrony with the tactile stimuli.

\section{SyStem PERformanCE}

\section{A. General Features}

Table I summarizes the key features of the device. The maximal stimulation range that can be achieved by the system is limited by the length of the rail of the linear

a)

b)

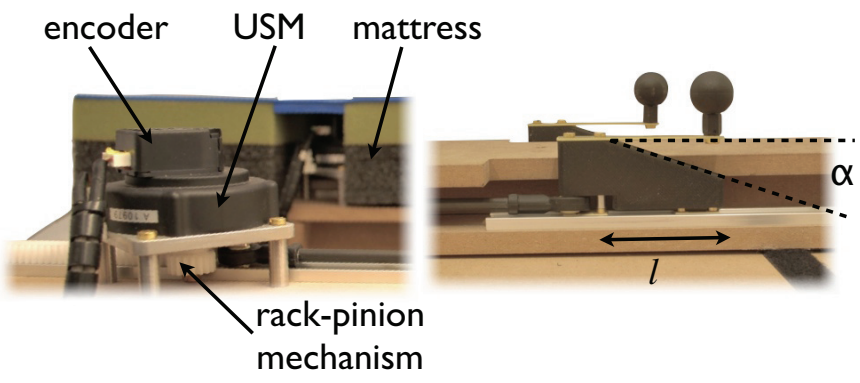

Fig. 4: The stroking mechanism consists of a USM motor that controls linear motion of a stimulation module through a rack-and-pinion gear. The stimulation sphere is attached over a flexible hinge to assure constant contact with the participant's legs or back. transmission. The external dimensions of the system are $175 \times 56 \times 20 \mathrm{~cm}^{3}$, which allows the placement of the system inside the scanner bore (Fig. 3) without affecting the height of the trunk and head over the scanner bed.

TABLE I: Specifications

\begin{tabular}{|l||c|}
\hline max. stroke back modules & $20 \mathrm{~cm}$ \\
max. stroke leg modules & $16 \mathrm{~cm}$ \\
\hline max. stroking velocity & $15 \mathrm{~cm} / \mathrm{s}$ \\
min. stroking velocity & $1.5 \mathrm{~cm} / \mathrm{s}$ \\
\hline control frequency & $200 \mathrm{~Hz}$ \\
display update rate & $40 \mathrm{~Hz}$ \\
\hline
\end{tabular}

The main program controls the position of the four modules during the stroking task. The position of each module is measured by the electro-optical encoder integrated with each of the ultrasonic motors. The control program runs at a sampling frequency of $200 \mathrm{~Hz}$, which is sufficiently high for the desired positioning tasks. The visual loop displays the real or manipulated position of the virtual stroking modules, and is updated at $40 \mathrm{~Hz}$.

\section{B. Dynamic Behavior}

The dynamic behavior of the system is limited by the USM (Shinsei, USR60-E3N, Japan). This piezoelectric actuator presents a dead zone below $15 \mathrm{rpm}$, and saturates at $150 \mathrm{rpm}$ [17]. In order to characterize the dynamic behavior of the unloaded system, a triangular position profile was commanded on the motor in PID position control. The position was measured with the encoder placed on the USM (Avago, HEDS-5545, USA) and converted to linear position 
considering the gear radius of $9 \mathrm{~mm}$. The frequency of the triangular position profile was varied from $0.05 \mathrm{~Hz}$ to $0.5 \mathrm{~Hz}$ in steps of $0.05 \mathrm{~Hz}$. Data were acquired over $60 \mathrm{~s}$ for each frequency.

Fig. 5 illustrates the behavior for a triangular signal at $0.4 \mathrm{~Hz}$ imposed on all four modules. The right BM (dashed line) presents a slightly shorter delay due to its mirrored mechanical configuration with respect to the three other modules. This can easily be compensated by software. As the position of the output is estimated from the position of the motor encoder, a second test was performed to characterize this relation with an external optical tracking system (accuTrack 500, atracsys, Switzerland). Despite the slight backlash in the rack-and-pinion gear, a good correlation (0.997) was found between the motor angle and output position, more than sufficient for the envisaged application.

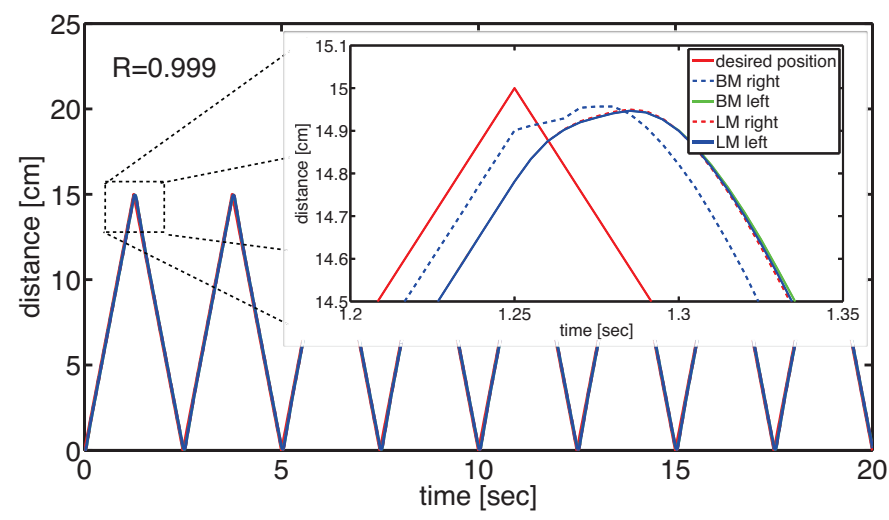

Fig. 5: Trajectories of the four stroking modules following a sawtooth position profile with an amplitude of $15 \mathrm{~cm}$ at a frequency of $0.4 \mathrm{~Hz}$. Three modules show an almost identical response, while one module has a slightly shorter delay due to the mirrored mechanical configuration compared to the three other modules.

\section{MRI Compatibility}

The compatibility of the system is determined by the actuation system, consisting of four ultrasonic motors placed close to the MRI bore, and the materials used. In the present system, only wood, polymers, brass and aluminum were used. Electrical power and data are transmitted from the control room to the MR room over shielded cables with RF filters at the level of the penetration panel and fiber optical links for the initialization of the modules.

Compatibility measurements were first performed on a $3 T$ Siemens Trio system and then repeated on a Siemens Magnetom $7 T$ (Siemens Medical, Germany) scanner using a 8-channel Tx/Rx RF-coil (Rapid Biomedical, Germany) and a $7.3 l$ spherical dimethylpolysiloxan oil phantom. Field maps (30 slices, slice thickness $2 \mathrm{~mm}$, gap $5 \mathrm{~mm}$, FoV $210 \times 210 \mathrm{~mm}^{2}$, matrix size $160 \times 160$ voxels, TR $2500 \mathrm{~ms}$, TE $27 \mathrm{~ms}$, FA $80 \mathrm{deg}$ ) were acquired before and after installing the device in the scanner room.

A second experiment consisted of 6 alternating blocks: three blocks of $50 \mathrm{sec}$ duration with the motors powered, running at a constant speed of $15 \mathrm{~cm} / \mathrm{sec}$; and three blocks of $50 \mathrm{sec}$ length, with the motors silent. Fig. 6 shows a phantom image from each of the two conditions as well as the subtraction of the two, which shows no disturbance from the actuated system.

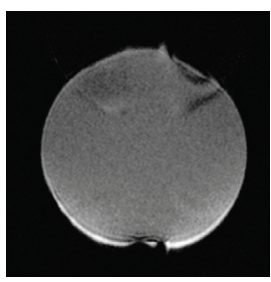

(a)

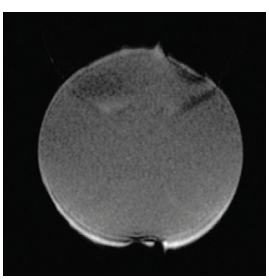

(b)

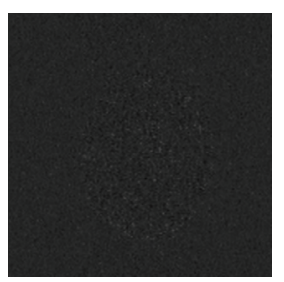

(c)
Fig. 6: Phantom scans from a 7 Tesla MR system with the interface at rest (left) and moving at constant speed of $15 \mathrm{~cm} / \mathrm{s}$ (center). The subtraction (right, high contrast) shows neither shifts nor deformations.

Further, we compared the variance of the signal-to-noise ratio (SNR) between the silent and powered conditions, in which the motor was driven at constant velocity or turned off, respectively. A two-sampled t-test revealed no significant difference between the SNR time series of the two conditions $(\mathrm{p}=0.56)$.

\section{Preliminary STUdy}

A simplified version of the presented device, consisting of a single stroking module placed behind the participants' back (BM), was used in a pilot study in combination with virtual reality and fMRI to measure brain activity during experimentally induced changes in self-location in 12 healthy participants (aged 21-26). Participants lay on the robotic stimulator that replaced the mattress on the MR bed. MRcompatible goggles showed the video of a stranger's back (or of an empty room) being stroked by a wooden stick with a spherical endpoint which resembled the stroking rod of the robotic device (visual input). At the same time the robotic device stroked the participant's back (tactile input). Direction and speed of the robotic stroking corresponded (synchronous) or differed (asynchronous) from the visual stroking. The study consisted of different blocks composed of video clips and movement profiles of the robotic stimulator grouped in four conditions according to a $2 \times 2$ factorial design with object (body, no body) and synchrony (synchronous, asynchronous) as main factors.

After the visuo-tactile stimulation, participants' selflocation was evaluated by using a mental imagery task: the "mental ball dropping" (MBD) [2], [4]. According to the procedure of the MBD, participants imagined releasing a ball they were holding in their hand, and estimated the falling time to the ground by pressing a button upon imagined impact. In the body conditions, response times for the mental ball dropping were significantly longer in the synchronous with respect to the asynchronous stroking condition, suggesting an elevation in self-location. This was not the case in the no-body conditions. fMRI results showed 
bilateral activation of the temporoparietal junction and precuneus with a significantly different BOLD signal change in the synchronous/body condition with respect to the other conditions, thus suggesting that the magnitude of activity in the temporoparietal junction, as manipulated through visuotactile conflicts, reflects the drift-related changes in selflocalization (Fig. 7). Other activated regions included the sensorimotor and supplementary motor areas.
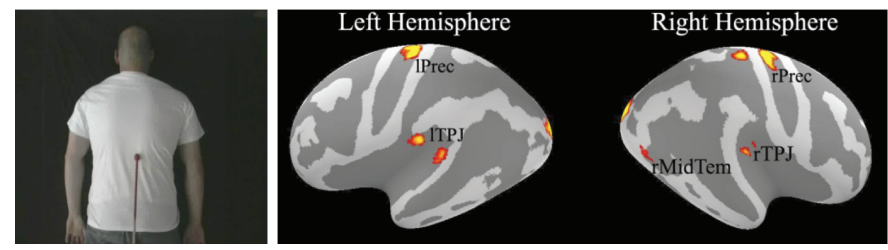

Fig. 7: Visual feedback (left) and fMRI results (right) of the preliminary study. The temporoparietal junction and precuneus showed significantly higher activation when visual and tactile stimuli were presented in synchrony.

\section{CONCLUSIONS}

This paper presented a novel robotic and virtual reality interface capable of applying multisensory stimuli to induce changes in self-location and investigate the neural correlates with fMRI. The design of the MRI-compatible device takes into account MRI safety and compatibility issues, as well as workspace and ergonomics constraints. The system consists of four individual stimulation modules actuated by four ultrasonic motors in PID position control. Visual feedback is provided through an MRI-compatible head mounted display, presenting virtual stimulation points overlaid on a picture of a body seen from the back. This approach gives full control over the visual feedback, and allows to introduce and modulate delays between the visual and tactile stimuli. System performance was evaluated by imposing the same triangular position profile at $0.4 \mathrm{~Hz}$ to all four modules, and showed a good synchronization between the modules. Compatibility tests with the complete system were performed on both a $3 T$ and $7 T$ MRI system, showing no significant effect on the MR imaging by the presence and motion of the system. While the pilot study used only simple visuo-tactile stimulation that mimicked previously used, nonrobotic patterns of stimulation, this novel robotic platform will allow us to test many other combinations of visuotactile stimuli, and to manipulate these in various manners. This promises novel insights into the neural underpinnings of multisensory integration and bodily awareness in health and neurological disease.

\section{ACKNOWLEDGMENTS}

This work was supported in part by a private foundation, the Center for Biomedical Imaging (CIBM) of the Geneva and Lausanne Universities, EPFL, and the Leenaards and Louis-Jeantet foundations. D. Chapuis and R. Gassert were partially supported by the National Center of Competence in Research on Neural Plasticity and Repair of the Swiss
National Science Foundation. The authors would like to thank José Marques and the staff of the Centre d'Imagerie BioMédicale for the valuable support during the compatibility tests.

\section{REFERENCES}

[1] O. Blanke, T. Landis, L. Spinelli, and M. Seeck, "Out-of-body experience and autoscopy of neurological origin," Brain, vol. 127, no. Pt 2, pp. 243-258, Feb 2004.

[2] B. Lenggenhager, M. Mouthon, and O. Blanke, "Spatial aspects of bodily self-consciousness," Consciousness and Cognition, vol. 18, no. 1 , pp. $110-117,2009$.

[3] M. Botvinick and J. Cohen, "Rubber hands 'feel' touch that eyes see," Nature, vol. 391, no. 6669, pp. 756-756, Feb 1998.

[4] B. Lenggenhager, T. Tadi, T. Metzinger, and O. Blanke, "Video ergo sum: manipulating bodily self-consciousness," Science, vol. 317, no. 5841, pp. 1096-9, Aug 2007.

[5] H. H. Ehrsson, "The experimental induction of out-of-body experiences," Science, vol. 317, no. 5841, p. 1048, Aug 2007.

[6] H. H. Ehrsson, C. Spence, and R. E. Passingham, "That's my hand! activity in premotor cortex reflects feeling of ownership of a limb," Science, vol. 305, no. 5685, pp. 875-7, Aug 2004.

[7] M. Tsakiris, M. Costantini, and P. Haggard, "The role of the right temporo-parietal junction in maintaining a coherent sense of one's body," Neuropsychologia, vol. 46, no. 12, pp. 3014-8, Oct 2008.

[8] O. Blanke and R. Gassert, "Total control in virtual reality and robotics," in Frontiers in Neuroscience, vol. 3, no. 1, 2009, pp. 110111.

[9] R. Gassert, R. Moser, E. Burdet, and H. Bleuler, "Mri/fmri-compatible robotic system with force feedback for interaction with human motion," Mechatronics, IEEE/ASME Transactions on, vol. 11, no. 2, pp. $216-224,2006$.

[10] R. Gassert, E. Burdet, and K. Chinzei, "Opportunities and Challenges in MR-Compatible Robotics," IEEE Engineering in Medicine and Biology Magazine, vol. 27, no. 3, pp. 15-22, 2008.

[11] J. Duenas, O. Lambercy, D. Chapuis, and R. Gassert, "Regrasp, a robotic tool to investigate fine motor control and track therapyinduced neuroplasticity," in Robotics and Automation (ICRA), 2010 IEEE International Conference on, May 2010, pp. 5084 -5089.

[12] S. Ionta, L. Heydrich, B. Lenggenhager, M. Mouthon, E. Fornari, D. Chapuis, R. Gassert, and O. Blanke, "Multisensory mechanisms in temporo-parietal cortex support self-consciousness," in press, 2011.

[13] R. Gassert, E. Burdet, and K. Chinzei, "MRI-compatible robotics," IEEE Engineering in Medicine and Biology Magazine, vol. 27, no. 3, pp. 12-14, 2008.

[14] R. Gassert, A. Yamamoto, D. Chapuis, L. Dovat, H. Bleuler, and E. Burdet, "Actuation methods for applications in MR environments," Concepts in Magnetic Resonance Part B: Magnetic Resonance Engineering, vol. 29B, no. 4, pp. 191-209, October 2006.

[15] B. Lenggenhager, P. Halje, and O. Blanke, "Alpha band oscillations correlate with illusory self-location induced by virtual reality," European Journal of Neuroscience, vol. 33, no. 10, pp. 1935-1943, 2011.

[16] M. Tsakiris, "My body in the brain: A neurocognitive model of bodyownership," Neuropsychologia, vol. 48, no. 3, pp. 703 - 712, 2010.

[17] "Shinsei Corporation Inc., USR60 series ultrasonic motor," http://www.shinsei-motor.com/English/welcome.html. 